\title{
Téoros
}

Revue de recherche en tourisme

\section{Les limites du tourisme cynégétique : des enjeux identitaires sous-jacents à la maîtrise des territoires}

\section{Le cas de la France}

\section{Christophe Baticle}

Volume 32, numéro 1, 2013

Tourisme cynégétique et halieutique

URI : https://id.erudit.org/iderudit/1036649ar

DOI : https://doi.org/10.7202/1036649ar

Aller au sommaire du numéro

Éditeur(s)

Université du Québec à Montréal

ISSN

0712-8657 (imprimé)

1923-2705 (numérique)

Découvrir la revue

Citer cet article

Baticle, C. (2013). Les limites du tourisme cynégétique : des enjeux identitaires sous-jacents à la maîtrise des territoires : le cas de la France. Téoros, 32(1), 7-16. https://doi.org/10.7202/1036649ar
Résumé de l'article

La mise en tourisme d'un loisir procède d'une opération qui achoppe parfois sur des résistances. Celles-ci relèvent des intérêts en jeu, comme l'appropriation du territoire entre gens du milieu et visiteurs, mais elles s'arment également des règles juridiques encadrant les pratiques, lesquelles témoignent des luttes menées pour faire advenir une forme de légitimité en faveur de certains groupes. La chasse apparaît, à ce titre, comme une illustration de milieux socialement hétérogènes, mais réunis dans une compétition pour faire prévaloir une conception anthropocentrée de la nature. À partir d'une étude socio-anthropologique des territoires, menée pendant près d'une dizaine d'années, cet article étudie le lien entre tourisme et chasse sur le mode du pourquoi. Qu'est-ce qui pourrait amener au développement du tourisme cynégétique, alors que cette activité de prédation s'inscrit dans une relation étroite avec des espaces vécus comme relevant d'une sphère de préemption pour les gens du lieu?

Pour illustrer ce propos, le cas de la France est particulièrement intéressant, puisque s'y ajoute un accent localiste. Se prévalant d'une " authenticité " enracinée qu'ils opposent schématiquement au consumérisme touristique, les chasseurs y ont néanmoins réussi à gérer la demande émanant de leurs collègues nomades, des touristes en puissance à bien des égards. Ils résolvent le dilemme par des formes de cooptations.

L'étude explique que l'enjeu se situe autour de la réticence des deux principaux groupes de pratiquants, propriétaires fonciers et chasseurs sans terre (mais locaux), à l'égard de toute commercialisation des territoires de chasse en direction des allochtones. Le tourisme devient alors l'antonyme de l'identité territoriale.
Ce document est protégé par la loi sur le droit d'auteur. L'utilisation des services d’Érudit (y compris la reproduction) est assujettie à sa politique d'utilisation que vous pouvez consulter en ligne.

https://apropos.erudit.org/fr/usagers/politique-dutilisation/ 


\title{
Les limites du tourisme cynégétique : des enjeux identitaires sous-jacents à la maîtrise des territoires Le cas de la France
}

\author{
Christophe BATICLE, post-doctorant \\ Socio-anthropologue \\ Université de Picardie Jules Verne (France) \\ cbaticle@aol.com
}

\begin{abstract}
RÉSUMÉ: La mise en tourisme d'un loisir procède d'une opération qui achoppe parfois sur des résistances. Celles-ci relèvent des intérêts en jeu, comme l'appropriation du territoire entre gens du milieu et visiteurs, mais elles s'arment également des règles juridiques encadrant les pratiques, lesquelles témoignent des luttes menées pour faire advenir une forme de légitimité en faveur de certains groupes. La chasse apparaît, à ce titre, comme une illustration de milieux socialement hétérogènes, mais réunis dans une compétition pour faire prévaloir une conception anthropocentrée de la nature.

À partir d'une étude socio-anthropologique des territoires, menée pendant près d'une dizaine d'années, cet article étudie le lien entre tourisme et chasse sur le mode du pourquoi. Qu'est-ce qui pourrait amener au développement du tourisme cynégétique, alors que cette activité de prédation s'inscrit dans une relation étroite avec des espaces vécus comme relevant d'une sphère de préemption pour les gens du lieu?

Pour illustrer ce propos, le cas de la France est particulièrement intéressant, puisque s'y ajoute un accent localiste. Se prévalant d'une «authenticité » enracinée qu'ils opposent schématiquement au consumérisme touristique, les chasseurs y ont néanmoins réussi à gérer la demande émanant de leurs collègues nomades, des touristes en puissance à bien des égards. Ils résolvent le dilemme par des formes de cooptations.

L'étude explique que l'enjeu se situe autour de la réticence des deux principaux groupes de pratiquants, propriétaires fonciers et chasseurs sans terre (mais locaux), à l'égard de toute commercialisation des territoires de chasse en direction des allochtones. Le tourisme devient alors l'antonyme de l'identité territoriale.
\end{abstract}

Mots-clés: Chasse, tourisme, France, préemption territoriale, identité.

«Rousseau refuse la clôture : à l'espace fermé de la propriété, il oppose le libre parcours d'une campagne ouverte. Cela vaut pour la chasse : «j’établirais mon séjour champêtre dans un pays où la chasse soit libre à tout le monde...»» (Larrère, 1988 : 42)

La rencontre entre tourisme et chasse a donné lieu à des images célèbres, notamment en Afrique, où les safaris offrent très tôt leurs services aux porteurs de fusils occidentaux (Daudet, 1872), mais encore en Europe de l'Est, avec un usage parfois diplomatique des chasses présidentielles pendant la période soviétique (Lejeune, 2005). Dans certaines régions du monde, comme l'Irlande, le tourisme cynégétique est considéré comme un levier du développement local (Scallan, 2008), alors que, dans d'autres, il reste quasiment absent, et ce bien que les conditions de son émergence semblent réunies. La France est à ce titre un cas de figure intéressant, dans la mesure où il s'agit d'un pays où la chasse joue un rôle considérable, $y$ compris sur les plans sociologique, politique et économique, sans que l'on puisse y observer un secteur touristique marchand au plein sens du terme. C'est là un constat d'autant plus surprenant qu'on se situe ici dans la première destination touristique du monde avec plus de 81 millions de visiteurs étrangers en 2011 (DGCIS, 2012).

En partant de ce hiatus, un questionnement s'impose quant aux conditions d'émergence d'un marché pour ce type de loisirs récréatifs, ici de nature, d'autant que ces derniers connaissent depuis plusieurs décennies un engouement indiscutable (Dalla Bernardina, 2011). Ce faisant, tout marché implique la rencontre entre une offre et une demande. C'est 
peut-être là le noud gordien de la problématique cynégétique pour la majeure partie du territoire français. Non seulement l'offre de chasse s'y révèle fort ténue mais, qui plus est, la demande s'exerce là sous des formes qui contredisent la logique marchande la plus commune (Widmer, 2008). En d'autres termes, on rechigne chez les porteurs de fusils à ce qu'une pratique investie d'une signification identitaire (LeroyWarnier et Caron, 2008) se retrouve régulée selon des modes de tractations principalement monétaires. Non pas qu'il n'y ait une réelle économie de la chasse sur la partie occidentale du continent européen, ni même que n'y seraient absentes des modalités d'échange éminemment pécuniaires, mais dans la mesure où la relation à cet exercice prédateur pose un principe préalable qui en fonde l'éthique.

Chasser s'entend comme une démarche de captation des ressources naturelles, dont la légitimité n'est recevable qu'en contrepartie à un investissement sur le long terme, en direction d'un territoire sur lequel on peut faire valoir un capital d'autochtonie (Renahy, 2005, 2010), toujours déterminant de façon tacite. Ce «capital» (un concept développé en France et trouvant son inspiration dans le cadre théorique initié par Pierre Bourdieu), vise à décrire le mode de légitimation qui prévaut dans les groupes de résidents plaçant au premier plan, parfois avec une très grande flexibilité, leur date d'arrivée sur le territoire. Généralement dominés sur la scène socio-économique, ils se réservent ainsi des sphères qu'on peut considérer comme secondaires (Chamboredon, 1982), mais essentielles pour le maintien de cette distinction établie avec les nouveaux arrivants, surtout si ces derniers les surplombent par leur dotation sur le plan économique ou culturel. Ces sphères, comme la captation du droit de chasser, qui revient finalement au contrôle des corps autorisés à s'approprier le territoire par sa faune sauvage, leur permettent de mener une existence sociale reconnue par l'interconnaissance qui règne dans la localité. Les travaux menés sur la question (Ripoll et Tissot, 2010) montrent, en effet, que le capital d'autochtonie vient souvent contrebalancer des phénomènes de dépendance dans d'autres secteurs de la vie en société, comme le champ professionnel. En ce sens, le capital d'autochtonie se caractérise par son efficience symbolique, mais il constitue également un capital social, autrement dit un carnet d'adresses mobilisables.

À défaut de disposer de cette «ressource sociale» (Retière, 2003), les impétrants se prévalent de l'entremise d'ayants droit, qui se portent garants pour eux quant à leur dignité à entrer dans l'espace électif des pérégrins. Cette opération de triage sociogéographique est parfois sanctionnée par une procédure de parrainage. Il subsiste en effet, dans la culture française, un très vieux fond ruraliste (Barral, 1968), par lequel persiste une forme d'ennoblissement ressenti au travers de la déambulation cynégétique. Au sein de ce cercle très fermé, on accède ainsi à une forme d'entre-soi localiste qui n'est pas sans expliquer la revendication à être chasseur, dont témoignent ces ouvriers d'origine rurale en phase de déclassement social, lesquels constituent l'armature sociologique de la chasse française contemporaine.

De la sorte, si Alain Corbin (2009) voit dans l'avènement des loisirs pendant l'entre-deux-guerres un moyen privilégié de détourner les ravages de la grande crise des années 1930 pour les classes populaires, on peut affirmer que la seconde démocratisation de la chasse correspond, en France, à un phénomène compensatoire de l'exode rural des années 1950 et 1960 (Chamboredon, 1982). À la différence qu'ici, le retour saisonnier au village a relevé d'une sélectivité en matière de réseaux d'interconnaissance, privilégiant d'autant mieux les natifs et leurs descendants qu'ils disposaient des codes sociaux à même de leur faire apprécier cette faveur accordée dans l'autorisation à pérégriner sur le finage.

L'analyse des ratés, dont la commercialisation des territoires de chasse a été l'objet, passe de ce fait par l'histoire des usages symboliques de l'espace rural, mais encore par le droit de chasse, intrinsèquement associé en France à la propriété foncière. Enfin, parce que les résistances se sont concentrées sur la question territoriale, dont la maîtrise reste souvent l'apanage du groupe installé, il convient d'en décrypter les ressorts. Véritable verrou interdisant l'ouverture à l'échange marchand, la collectivisation des terres, sur le plan cynégétique, procède de la pression des chasseurs à l'égard des détenteurs du droit de chasse. C'est par le biais de leur reconnaissance locale à s'inscrire dans une "tradition» que le capital foncier est amené à composer avec le capital d'autochtonie, lequel fait valoir le droit à chasser pour les résidants et natifs face au droit de chasse détenu par les propriétaires. On comprend mieux alors l'enjeu dont recèle l'attitude fort distanciée à toute marchandisation des "plaisirs de la chasse» sur le mode de ce que l'on connaît pour d'autres services en matière de loisirs de nature. Il y a là aussi la marque d'une société française qui continue à établir une relation éminemment emblématique avec la terre (Chevallier, 2000). C'est d'autant plus vrai que l'on connaît ici, depuis le milieu des années 1980, un renouveau du ruralisme qui fit de la France l'un des premiers pays agrariens d'Europe (Robert, 1986). Si on a ainsi pu penser que la chasse n'échapperait pas à la libéralisation des loisirs et à son absorption par le capitalisme, il n'en est encore rien ici. De la même manière, la généralisation du «modèle anglais», pronostiquée par Marx (1850) pour l'agriculture, buta sur la structuration familiale de l'exploitation agricole, laissant le grand capital aux portes des fermes. Même de nos jours, la capitalisation en grandes firmes reste une tendance parmi d'autres (Hervieu et Purseigle, 2013).

Plutôt donc que d'étudier la connexion entre tourisme et chasse sur le mode du comment, il est envisageable de se demander pourquoi les pratiquants se montrent si peu enclins à voir apparaître ce segment commercial, tandis que certains parmi eux peinent à trouver un territoire d'exercice? Si les services existants ne connaissent qu'un succès limité, n'est-ce pas alors la nature de la demande qui ne peut s'en satisfaire? Il conviendra, dans un premier temps, de valider cette faible tonicité du tourisme de chasse (Actéon, 1998), avant d'en aborder les deux principaux freins, l'un s'attestant dans la régulation juridique du droit de chasse, produit d'une longue histoire de luttes entre propriété foncière et droit d'usage, l'autre relevant de la symbolique territoriale. Cette problématique s'inscrit dans le cadre d'une socio-anthropologie des territoires, menée pendant près d'une dizaine d'années, et qui a abouti à une thèse tirant parti de vastes enquêtes par questionnaires, d'entretiens et d'une immersion par observation participante. 


\section{Quid d'un marché du tourisme cynégétique en France}

Paradoxalement, alors que la France constitue la première clientèle européenne avec encore 1331000 validations ouvrant droit à la pratique (FACE, 2010), on y constate un accès aux territoires passant rarement par le marché. Même si nous sommes très loin du point d'apnée atteint en 1975 avec 2,2 millions de chasseurs, Xavier Patier (2002: 15) observe que la diminution des effectifs est ici moins importante que dans la majeure partie des États du vieux continent. En 2009, la densité cynégétique n'était que de 1,33 \% au sein de la Communauté européenne, contre le double en France (Univers Nature, 2009). Toutefois, cette cynégéphilie (intérêt pour la chasse) ne s'arrête pas là, le poids des chasseurs dans la société française allant jusqu'à faire du groupe d'étude spécialisé de l'Assemblée nationale l'un des tout premiers : 154 membres en mai 2012. Son impact s'illustre ainsi essentiellement par ses dimensions sociétale et politique.

Pour autant, l'économie de la chasse pèse localement d'un poids non négligeable dans la valeur ajoutée issue des activités de loisirs (Vollet et Bretière, 2008; Le Goffe et Vollet, 2008; IFE, 1999). Notons néanmoins qu'il s'agit ici d'un sujet soumis à des enjeux hautement politiques. On l'observe avec l'insistance des édiles cynégétiques à avancer cet argument dans les périodes paroxystiques de crise de légitimité de la pratique. Reste que ce qu'il en ressort touche au chasseur consommateur de biens et de services, mais peu à la commercialisation des territoires au travers de marchés véritables, ouverts à l'accueil de chasseurs extérieurs (chasses à la journée, séjours).

Se pose ainsi un problème de taille pour évaluer l'impact du tourisme cynégétique, laquelle difficulté renvoie finalement à la définition adoptée pour qualifier le phénomène touristique lui-même. Pour l'Organisation mondiale du tourisme, la démarche consiste en "un déplacement hors de son lieu de résidence habituel pour plus de 24 heures mais moins de 4 mois, dans un but de loisirs, un but professionnel (tourisme d'affaires) ou un but sanitaire (tourisme de santé)» (MAE, 2003 : 8). Pour être au plus proche de la réalité cynégétique, il conviendrait d'intégrer les excursions inférieures à 24 heures et alors proposer le cadrage suivant : sortie monnayée pour motif de chasse sur un territoire non détenu en propriété et en dehors des associations communales de sa ou ses communes de résidence. Cette délimitation permettrait de pallier les caractéristiques inhérentes à la pratique, à savoir, d'une part, la liaison entre propriété et droit de chasse et, d'autre part, l'affiliation courante des résidents, insérés de facto au sein des associations locales. Mais en réalité, l'accession à ces «sociétés communales» présente d'autres biais, comme ceux ouverts par les règlements intérieurs avec la possibilité offerte aux parents non-résidents ou encore par les cartes d'invitation gratuites à la journée, laissées à la discrétion des sociétaires. On le perçoit, la configuration de la chasse nécessite de déterminer ce que l'on cherche à cerner. Retenir la définition officielle du tourisme amènerait à minimaliser le poids du commerce induit autour des chasses commerciales à la journée et à le réduire aux voyages cynégétiques. À l'inverse, ouvrir le périmètre conduirait souvent à considérer comme touriste le fils invité pour un dimanche dans son village natal, alors qu'il en est le voisin proche. Le problème est, semble-t-il, ailleurs.
Tout d'abord, une économie touristique implique la nonsélectivité des consommateurs, donc un marché en bonne et due forme. Est-on véritablement touriste si l'on vient réguler les espèces occasionnant des dégâts dans les propriétés voisines des siennes, ou si l'on dispose d'une carte dans une société communale au titre des surfaces foncières que l'on apporte au fond chassable? Or, une difficulté centrale est avérée sur ce point, puisque seules les chasses commerciales procèdent sur le mode marchand, dans une approche libérale. Néanmoins, les consommateurs de ces espaces libéralisés ne sont pas nécessairement, et ici encore, des touristes. Ils peuvent relever d'une pratique occasionnelle de grande proximité en l'absence de territoire pour certains chasseurs du secteur, qui compensent par là leur déficit en capital local, que ce dernier ressorte du relationnel ou de la détention foncière. En d'autres termes, bien que l'on ait inauguré la catégorie du «tourisme de proximité» (Goetschmann, 2010) pour pallier la question sous-jacente de la distance, celle-ci reste prégnante. N'est pas touriste le consommateur à domicile. Pourtant, en matière de chasse, il existe bien d'autres manières d'être chez soi ailleurs que là où l'on réside. C'est même l'un des ressorts essentiels de l'intégration à la plupart des territoires.

Pour ces raisons, une approche par les dépenses d'hôtellerie ne permet pas davantage de cerner le public touristique, bien qu'il résolve grandement la question de la distance inhérente à la conception que l'on se fait du touriste. D'autre part, les chasseurs usagers des structures d'hébergement n'en sont pas indubitablement des consommateurs, au sens où ils n'auraient d'autres comptes à rendre que le respect du règlement intérieur. Le fait de devoir s'acquitter d'un droit d'accès ne signifie souvent en rien que l'on soit acceptable pour déambuler fusil en main. Il s'agit toujours, dans le contrat implicite entre l'impétrant et le groupe intégrateur, de fournir les gages d'un savoir-être en phase avec le système de valeurs en vigueur localement, ce qui n'exposerait pas le touriste à se trouver exclu de droit s'il y contrevenait. Autant de motifs qui amènent à remettre en question la pertinence du terme «tourisme» lorsqu'il s'applique à un champ aussi spécifiquement peu ouvert que celui de la chasse, territoires et même gibiers n'étant pas, contrairement à ce que la législation affirme pour ces derniers, des «biens sans maître », loin de là. Même lorsque l'espace n'est pas détenu en propriété par les porteurs de fusils, ces derniers ne s'en considèrent pas moins préempteurs des ressources faunistiques au travers de leurs aménagements du territoire, du nourrissage du gibier, voire du repeuplement.

Ainsi, et de façon révélatrice, l'entrée "tourisme cynégétique» n'a pas été retenue comme spécialement significative dans les deux grandes enquêtes dont on dispose au niveau national (Pinet, 1987, 1993). Primo, elle n'y est qu'une dimension marginale. Secundo, dans la mesure où elle traite des voyages de chasse à l'étranger réalisés par des fusils nationaux, lesquels déplacements ne représentaient par ailleurs que 1,3\% des flux financiers générés par la chasse française en 1986 et $1,2 \%$ en 1992, induits par respectivement $2,6 \%$ et $2,5 \%$ des adeptes du pays. De plus, seules de rares données fragmentaires traitent du tourisme intrahexagonal, et ce indirectement par les dépenses d'hôtellerie et de déplacement vers les sites d'exercice. En revanche, ce que l'on pourrait considérer comme l'indicateur 


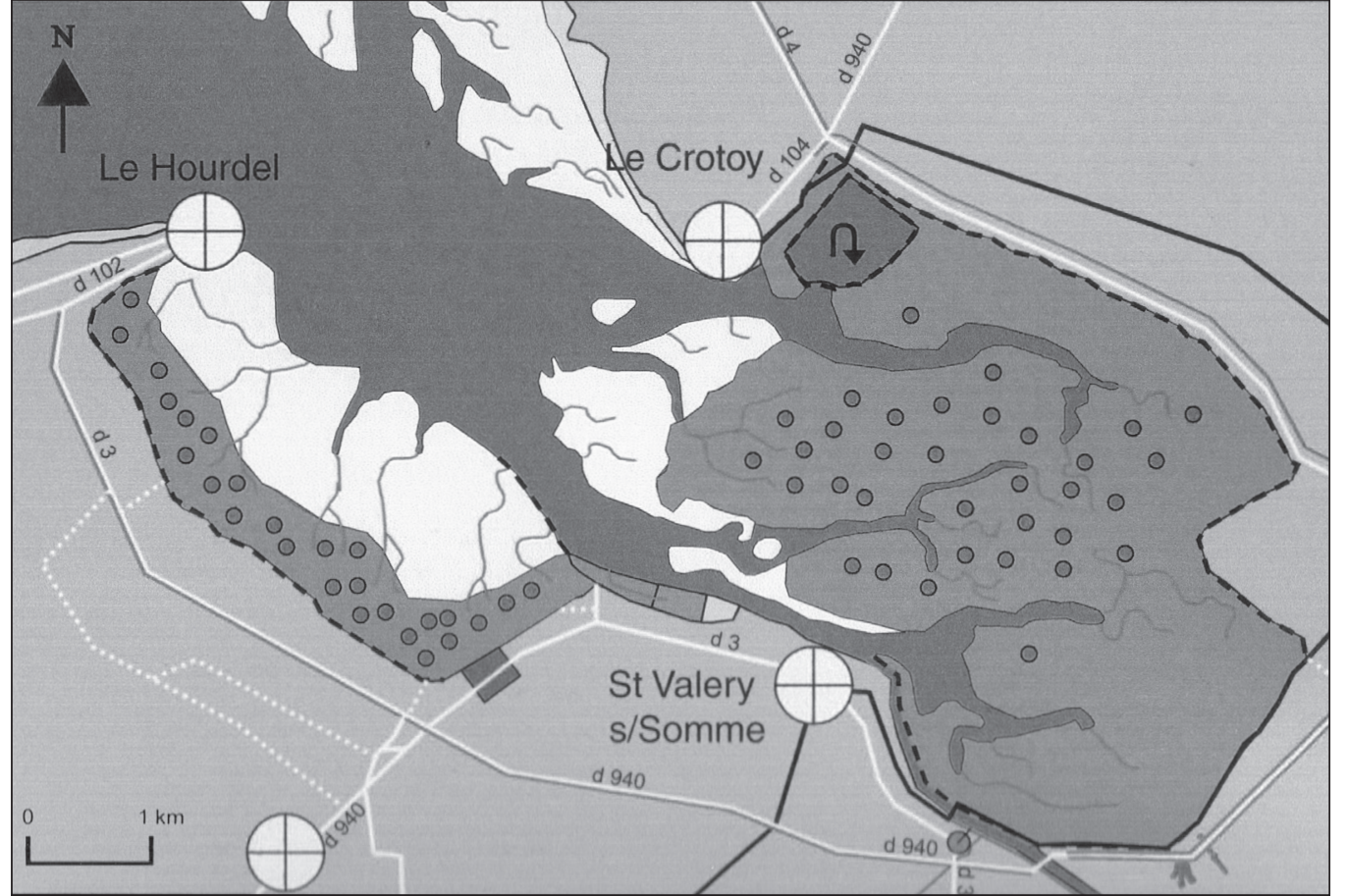

ILLUSTRATION 1 : Densité des huttes de chasse en baie de Somme (159 installations) (source : Sannier (2004: 45)). déterminant, soit le chiffre d'affaires des pourvoyeurs de chasse commerciale, ne transparaît tout simplement pas en 1986, alors qu’il est évoqué à la marge six ans plus tard (Pinet, 1993 : 54).

En 1992 donc, le statut de "société de chasse privée à but commercial» apparaît bien isolé parmi les quelque 30000 associations à but non lucratif que compte la chasse française, et on ne se risque d'ailleurs pas à en fournir un effectif. On indique que $12,5 \%$ des pratiquants y avaient eu recours, pour une moyenne annuelle de 3,6 journées par individu, générant à l'époque un peu plus de 730000 sorties individuelles et 95 millions d'euros actuels (Pinet, 1993 : 54). S'il s'agit à première vue d'un marché substantiel, il ne représentait que $1,5 \%$ des sorties, mais près du quart des coûts d'accès aux territoires, ce qui revient à dire que ces chasses à la journée s'avéraient spécialement onéreuses comparées aux formes associatives communales, très abordables au contraire. Cet aspect financier contribue par ailleurs à expliquer le faible développement de ce négoce, mais surtout à éclairer la propension limitée pour ce type d'offre.

Sur le plan des activités générées, en posant l'hypothèse d'un chiffre d'affaires de 105000 euros contemporains par emploi, Pinet (1993: 92) en arrivait à estimer à 950 les Français tirant leur travail de cette marchandisation des territoires, soit seulement $4 \%$ du secteur de la chasse. Là encore, il semblerait que la pratique cynégétique n'ait constitué qu'un sous-secteur touristique très résiduel. Ainsi, sur une dépense annuelle moyenne par chasseur de 1660 euros, seuls moins de 20 relevaient des déplacements à l'étranger, soit un peu plus de $1 \%$ du budget, alors que 60 étaient dirigés vers les chasses commerciales intérieures (Pinet, 1993 : 88).

Sommes-nous alors devant un tourisme ignoré en tant que tel et, dans ce sens, les dépenses d'hébergement permettent-elles de révéler la partie immergée de l'iceberg? Il n'en est rien, les nuitées d'hôtellerie pesant en 1986 pour 17 millions d'euros, soit à peine plus d'un demi-point des dépenses induites par l'activité et guère qu'un dixième de plus en 1992 (Pinet, $1987: 61 ; 1993$ : 79). Ils sont alors moins de $5 \%$ à déclarer ce type de dépenses.

Subsiste néanmoins une exception à cette insoluble contradiction des faits : la chasse littorale sur le domaine public maritime, relevant des prérogatives de l'État. Depuis 1975 en effet, les espaces littoraux sont organisés sous le couvert de structures reconnues (les associations de chasse du domaine public maritime, ou ACDPM), qui contractualisent leur bail directement avec l'autorité publique. Ce sont là de puissantes organisations cohésives, qui proposent une conception traditionaliste de la nature et concentrent en leur sein les plus virulentes oppositions à toute dynamique ressentie comme exogène, dont le tourisme (Valicourt, 1972). Pour autant, dans les années 1980, elles ont vu leur attractivité redoubler à la faveur d'un mouvement en direction du gibier d'eau, corrélatif à l'appauvrissement de la giboyeusité (à savoir la densité de gibier sur un territoire) dans les plaines. Leurs adhérents, beaucoup plus jeunes que la moyenne et relevant davantage des classes populaires (Baticle, 2005), se répartissent en trois grandes composantes : d'abord, les résidents immédiatement locaux, issus des communes limitrophes du bord de mer, héritiers des pionniers des modes de chasse dévolus aux côtes; ensuite, les chasseurs régionaux, arrivés progressivement avec le développement des transports automobiles; puis, enfin, d'authentiques touristes, dans le sens où leur implication territoriale se restreint effectivement à une consommation sans implication laborieuse pour l'aménagement des espaces de chasse. 
ILLUSTRATION 2 : Rétraction des effectifs dans les associations de chasse du domaine public maritime picard (source : Baticle (2007:601)).

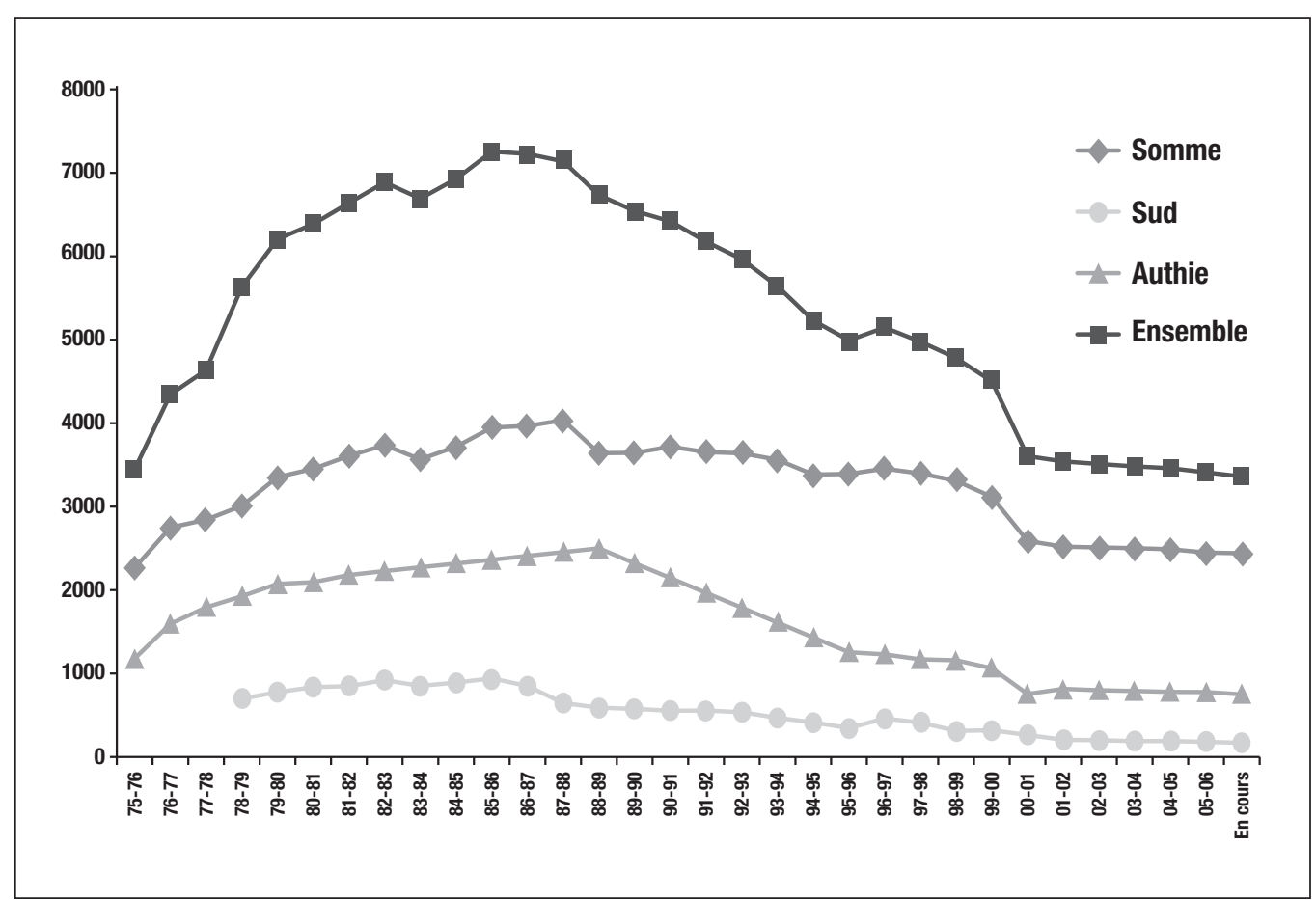

Haut lieu de la sauvagine s'il en est, le littoral picard fait figure d'emblème parmi les territoires acquis à la cause cynégétique, et ce, jusqu'au-delà de l'Atlantique. Marcel, guide cynégétique sur le lac Saint-Pierre, entre Montréal et Québec, lui-même chasseur de gibier d'eau, témoignait lors d'un entretien en 2010 de ce sentiment : «À chaque fois que je suis allé en baie de Somme, j'ai ressenti une ferveur... que je n'avais pas vue jusque-là. Comme s'ils étaient chasseurs avant tout.» Le mode de chasse qui s'y pratique prioritairement n'y est pas étranger, puisqu'il exige une implication constante pour maintenir l'attractivité du territoire : entretien des mares creusées dans les fonds vaseux, fauche des végétaux, gestion des niveaux d'eau.

On y distingue trois formes de pratique, soit la hutte, le hutteau et la botte. Le premier type consiste en une installation pérenne et spartiate permettant l'attente noctambule du gibier qui se déplace de nuit. Il est monopolisé par les chasseurs locaux, qui revendiquent la primo-installation de leurs aïeux, peu à peu rejoints par des chasseurs régionaux ayant fourni les preuves de leur adhésion à cette idée d'un site d'exception. Y accéder nécessite ainsi une longue incrustation temporelle, qui se résume par l'expression : «la clé de l'hutte est au bout de ch'louchet », ce qui revient à dire qu'il est exigé de l'impétrant de s'user les mains sur le manche de la bêche avant de pouvoir prétendre accéder à ces abris de fortune installés à même les baies et les marais. Le département de la Somme s'affiche comme le premier en France pour le nombre de huttes qui s'y trouvent : plus de 2200 sur quelque 14000 dans tout le pays (Baticle, $2004: 29$ ). La baie de Somme en est spécialement parsemée (Illustration 1). Quant au hutteau, il procède de la même technique, mais avec des gîtes encore plus rudimentaires, installés à partir d'une bâche ou d'un «cercueil» dans lesquels on se calfeutre pour la nuit. On y trouve les passionnés d'un rapport rugueux au milieu sauvage, parfois dans l'attente d'une place dans une hutte. Enfin, la «botte» relève d'une chasse devant soi de jour (à la billebaude), et c'est par son biais que se développa le tourisme cynégétique dans la pleine acception du terme. Lors des années 1990, on compta, pour l'association de la baie de Somme, des résidents de plus de 50 départements métropolitains, ainsi que des ressortissants belges, alors qu'ils n'étaient plus que 31 départements lors de la saison 2001-2002 (Baticle, 2003 : 251-254).

Contrairement, donc, à une assertion courante dans les milieux cynégétiques, une forme de chasse investie par les pratiquants extérieurs n'est plus ici d'actualité. Une étude plus fine sur les codes postaux confirme ce mouvement de désinvestissement. Tendance encore plus évidente pour l'association du littoral picard sud : ses effectifs sont passés de 943 en 1985-1986 à moins de 200 aujourd'hui (illustration 2). La principale raison expliquant cette désaffection chez les «chasseurs aux pieds nus» est le raccourcissement de la période estivale pour les limicoles. De ce fait, les touristes cynégètes y ont disparu. Mais ces derniers ont-ils dynamisé les effectifs jusqu'à une période récente? En baie d'Authie, par exemple, les chasseurs non limitrophes ont représenté jusqu'à $45 \%$ des licences émises en 1976-1977, donnant à cette association une image de «Paris-plage». Mais, leur diminution intervient dès la saison suivante, alors que la croissance d'ensemble se poursuit jusqu’à la fin des années 1980 (Baticle, 2007 : 593-596).

Dans leur ensemble, les trois associations de chasse du domaine public maritime du littoral picard ont perdu plus de 3500 membres depuis la fin des années 1980, un recul principalement imputable aux vacanciers, qui exerçaient une chasse à la botte bon marché et n'exigeant pas de 
recourir à l'entremise des chasseurs locaux, ni à l'investissement sur le long terme.

Cette configuration se retrouve sur l'ensemble du littoral français, qui voit ainsi une des formes les plus abouties de tourisme cynégétique se réduire considérablement. Pour l'ensemble des modes de chasse, l'apathie du marché trouve ses origines dans des causes plus structurelles, qui tiennent d'abord dans l'organisation du droit de chasse, faisant la part belle au droit de propriété.

\section{De multiples chassés-croisés dans l'histoire du droit de chasse}

En introduction de ce point portant sur les conditions d'émergence d'un marché cynégétique vu sous l'angle préliminaire de la législation spatiale, il est nécessaire de revenir sur les fausses évidences d'une logique historique en faveur de la libéralisation du droit de chasse. Dans cette perspective, non seulement la mise en tourisme de la prédation n'est pas une fin à déduire d'un sens de l'histoire (tout comme l'élevage n'a pas signé l'arrêt de mort de la chasse), mais s'intensifie au contraire une confrontation sur la définition des espèces pouvant entrer dans le cycle du produit. Entre, d'un côté, les animaux d'élevage, ceux relevant du domestique ou encore les animaux nuisibles qui peuvent relever de la sphère marchande et, d'un autre côté, les taxons protégés généralement interdits à toute vente, le gibier dispose d'un statut particulier : appropriable selon certaines règles, dont financières, mais très encadré quant à son commerce. Dans certains cas, cette option est totalement prohibée, mais c'est surtout sur l'aspect moral qu'elle est réprimandée (Bozon et al., 1981).

Néanmoins, le particularisme des animaux chassés constitue un phénomène plus séculaire. Pour illustration, certaines espèces animales, facilement domesticables et intéressantes sur le plan du rendement carné, ont été délibérément maintenues dans la catégorie des gibiers depuis le néolithique (Testart, 1988). Il n'y a donc pas à se surprendre que la chasse échoue à se présenter tel un «loisir comme un autre». Acte mortifère par essence, il continue à lui échoir le rôle de conservatoire culturel. Il est alors compréhensible que l'on n'ait pu légiférer sur la traque des animaux sauvages à la manière d'un simple produit. On assiste de ce fait à un antagonisme constant quant à la question des terrains admissibles à l'action de chasse, surtout depuis que se sont multipliés les zonages protecteurs. Là encore, il ne s'agit pas ici d'un fait nouveau, mais inscrit dans la longue durée. En remontant celle-ci, il est possible d'appréhender une part des résistances qui portent sur l'accessibilité à l'espace, et dont le tourisme cynégétique n'est qu'une dimension récente.

On observe, en Europe occidentale, une lutte séculaire entre héritiers d'un titre anoblissant et détenteurs d'un fief pour s'attribuer le monopole du droit à la prédation, et ce pendant tout le Moyen Âge jusqu'à la fin de l'Ancien Régime (Salvadori, 1996). L'administration de la nature au travers de la prédation contrôlée perdure d'ailleurs jusqu'à ce jour sous des formes renouvelées (Baticle, 2007 : 60-91). Autrement dit, au-delà du fait de savoir ce qui représenterait un écueil à des mouvements tendanciels, les sociologues relèvent que le résultat abouti a souvent eu pour effet de masquer les luttes intenses qui ont présidé à la bonne fortune d'une position sur d'autres.

Il faut ici rappeler qu'en France, la moindre parcelle de terrain est généralement la possession jalousement gardée d'une inscription familiale (Baticle, 2009). L'expression «chasse gardée», passée dans le langage courant, est assez révélatrice de la prééminence de ces conflits cynégétiques qui voient le propriétaire microparcellaire se réserver le droit de chasse à l'encoignure des grands domaines de chasse. Dans de nombreux départements, les fédérations des chasseurs en ont fait le problème central d'une organisation rationnelle des territoires. Cette dernière représente, à bien des égards, un préalable à la marchandisation de la traque, dans la mesure où le produit chassé nécessite, pour être attrayant, des conditions de giboyeusité qui passent par un territoire suffisamment spacieux et géré dans sa globalité. Or, la chronologie juridique fourmille d'exemples qui illustrent par quels chemins tortueux sont passés les textes légiférant la chasse. En arrièreplan, se dessinent des conflits de nature idéologique, qui sont encore le reflet d'affrontements politiques entre composantes sociales. Mais, ce que démontrent avant tout les lois françaises, c'est que, loin d'être guidées par un dessein linéaire, elles multiplièrent les allers-retours avec, néanmoins, une constante : l'enjeu central du territoire et de ses ayants droit légitimes.

Lors de la révolution bourgeoise de 1789, un tournant semblait adopté définitivement avec l'ouverture du droit de chasser au commun, rejetant dans un premier temps l'appropriation acquise par la noblesse. Très vite cependant, la dénonciation des ravages subis par le gibier fit opérer un revirement: on rattacha le droit de prélever la faune sauvage à la propriété foncière. C'était la fin d'un très court acquis révolutionnaire dont on fait encore aujourd'hui grand cas, alors qu'en réalité, le privilège variait d'une région à l'autre (Beck, 1988).

Malgré tout, la lente conquête paysanne de la chasse (Bart, 1988), par l'appropriation de son outil de travail, aboutit à nouveau au sentiment d'une certaine anarchie régnant dans les plaines. Pour y mettre un terme, advient en 1844 le véritable «temps des propriétaires» (Aucante et Aucante, 2004) avec la grande loi réaffirmant la propriété comme fondement juridique inaltérable, et surtout les forces de répression afin de le faire respecter. «Nul n'a le droit de chasser sur le terrain d'autrui sans son consentement préalable», stipule le texte (Berton et Gaillardon, 2013 : 65). En pratique, l'émiettement plus ou moins prononcé du parcellaire devient le régulateur de l'accès à des espaces chassables d'un seul tenant. Chaque possédant règne en maître sur son emprise foncière et se crée des obligés en permettant, de son libre arbitre, l'ouverture de ses territoires à qui bon lui semble. Une petite contre-révolution se trouve ainsi confortée. Elle n'ouvrira pas sur des débouchés touristiques, sauf sur quelques zones humides ou de déprise agricole, comme en Sologne pouilleuse (Aucante et Mirloup, 1979).

Un siècle plus tard, la mal-nommée "révolution nationale», qui entame la bureaucratisation du droit de chasser, confisque toute forme de chasse banale en instaurant l'obligation d'adhérer aux nouvelles fédérations départementales dont elle établit l'acte de naissance (Vivent, 2005). Mais conjointement, avec l'État français du Maréchal Philippe Pétain, 1941 résonne 
comme une étape décisive qui va paradoxalement redonner la main aux chasseurs sans terre, lesquels vont se saisir des nouvelles entités administratives pour se diriger vers des structures de type associatif et, ainsi, regagner du terrain sur les propriétaires. Peu à peu, le collectif des chasseurs regroupés devient la réalité centrale des relations intravillageoises, faisant contrepoids aux inclinations élitistes des notables territoriaux. Au sens de sa théorisation par Émile Durkheim (1894), on peut qualifier cette situation de véritable contrainte sociale, amenant les détenteurs de surfaces moindres ou morcelées à rejoindre la dénommée "société des chasseurs». Cependant, dans un cas de figure comme dans l'autre, qu'il s'agisse d'une organisation articulée sur les propriétaires ou sur les associés, la question du libre accès reste entière. Si les premiers procèdent par invitations auprès de leur réseau social, il n'en va pas autrement pour les seconds, qui visent ni plus ni moins qu'à entretenir un entre-soi local, élargi aux chasseurs originaires du lieu (Bages et Nevers, 1982; Bozon, 1982).

C'est tellement vrai que le sénateur socialiste Verdeille réussit, en 1964, à enfoncer un coin dans le pilier patrimonial en obligeant les petits propriétaires parcellaires à adhérer aux associations communales de chasse agréées (ACCA). Toutefois, cet avantage conféré aux fusils sans terre est assujetti à une contrepartie qui les astreint à intégrer au moins $10 \%$ de leurs collègues extérieurs à la localité. Bien que la plus grande partie méridionale des départements français adhère aux ACCA, la brèche territoriale reste modeste du fait des contournements offerts par la notion d' «extérieur».

Les points d'achoppement furent ainsi légion entre, d'une part, un droit de propriété à tendance réservataire et, d'autre part, les luttes populaires pour l'accès aux territoires. De nombreuses joutes juridiques ont vu s'affronter deux grands courants de pensée, l'un arcbouté sur le droit de propriété, tandis que l'autre visait à faire reconnaître un droit d'usage essentiellement acquis aux titulaires du capital social local. Mais, dans cette conflictualité structurante pour le champ cynégétique, la place que s'est octroyé le commerce du tir resta à la hauteur de l'étroitesse laissée pour cette troisième voie. Les entrepreneurs de chasse devaient se doter d'une denrée convoitée et dont la mainmise était déjà l'apanage de réseaux locaux bien établis. Même le régime dérogatoire et spécifique accordé à l'AlsaceMoselle n'a pas permis une mise en exploitation généralisée de la ressource faunistique sauvage. Dans ces trois départements français, perdure en effet la loi du 7 février 1881, qui attribue à la commune l'administration du droit de chasse pour le compte des petits propriétaires parcellaires (Malafosse, 1979). La plupart du temps, on y procède ainsi par adjudications, comme pour les domaines de l'État. Or, ce sont des groupes constitués de chasseurs qui se présentent et obtiennent l'espace chassable (Hell, 1985), n'ouvrant son usage cynégétique que sur cooptation ou recommandation, certes contre cotisation ou action, mais très loin de ce qui pourrait représenter l'embryon d'un marché touristique ouvert.

Ces obstacles juridiques déterminants pour la mise en tourisme de la chasse font cependant écho à des modes d'appréhension proprement sociaux et culturels des usages légitimes de la nature, laquelle appréhension passe en France par le truchement de la dimension territoriale.
Chasse versus tourisme : les terrains de la discorde Lorsque l'on pense aux conflits d'usages entre activités qui prennent le même territoire pour support d'exercice, des questions viennent à l'esprit quant aux possibilités de rendre compatibles des démarches différenciées qui se doivent de partager non seulement une surface, mais encore des modes d'action profondément marquants par l'empreinte anthropique dont ils estampillent le territoire. À ce titre, la chasse contribue à la qualification de microlieux, comme avec la Sologne au sud de Paris, le col de l'Escrinet en Ardèche, celui d'Iraty dans les Pyrénées, les zones humides des bassins d'Arcachon dans le Sud-ouest français ou de Camargue au sud-est, la Dombes au nord de Lyon, ou encore le Médoc sur l'estuaire de la Gironde et la Grande Brière sur celui de la Loire. Sur certains de ces terroirs, les instances cynégétiques ont elles-mêmes lancé une offre de services à destination des chasseurs nomades (Iraty, par exemple). Sur d'autres, l'opposition est beaucoup plus forte de la part des chasseurs de base. Les ouvriers des chantiers navals de la Loire continuent à tenir la Grande Brière (Mischi, 2008). Mais, une nouvelle donnée émerge : le tourisme hors chasse se diffuse et les tensions avec le monde cynégétique se radicalisent, amenant ce dernier à se retrancher derrière l' «authenticité» de ses «traditions». En conséquence, le touriste est mis au pilori pour ce qu'on lui attribue dans l'artificialisation des territoires.

Toutefois, un constat s'impose : agriculteurs, sylviculteurs, chasseurs et autres exploitants des ressources naturelles ne sont plus les seuls ayants droit, contestés dans cette prérogative par de nouveaux acteurs, dont les touristes, et surtout l'écotourisme, qui répond aux fonctionnalités nouvellement légitimées des campagnes en matière de préservation écologique et patrimoniale (Perrier-Cornet, 2002). En ce qui concerne la baie de Somme, Lancelot Sannier (2004) relève qu'il n'y aurait pas d'insoluble antagonisme sur le plan des usages en vigueur, dans la mesure où l'occupation doit s'envisager sur un mode spatio-temporel. Les cynégètes qui pratiquent «à la hutte»y sont essentiellement présents pendant les saisons froides et, qui plus est, dans une forme d'occupation nocturne, quand les esthètes admirent le paysage de jour et sous le soleil.

Le duel que se livrent les représentants des deux parties pour le monopole de la légitimité à pérégriner sur le territoire laisse penser que ce sont moins les conflits effectifs qui font tension que les représentations croisées dont ils s'affublent mutuellement. Pour preuve, à l'étiquette «baie des phoques", véritable aimant pour les visiteurs, les tenants d'une «baie des chasseurs» répondent par le jeu de mots «louphoque» (Salesse, 1993), qui signifie fantaisiste, tout en dénotant l'idée d'un outrage. En réponse, le 15 avril 2000, alors que se discutait à l'Assemblée nationale la grande loi sur la chasse qui renouvela une part substantielle du cadre réglementaire posé en 1844, des sauvaginiers (chasseurs de sauvagines) démontaient quelques tronçons de rails de la ligne ferroviaire ceinturant la partie terrestre de l'estuaire, empêchant ainsi le tortillard à vapeur de transporter ses premiers voyageurs de la saison estivale. Aux cris de "pas de chasse, pas de tourisme», les chasseurs se sont attaqués ce jour-là à l'un des plus éminents symboles du tourisme côtier au travers de ce "petit train de la baie de Somme», entièrement réhabilité dans les années 1980 en direction des seuls visiteurs vacanciers. 
Sur le terrain, s'opposent deux images d'un joyau entré, depuis quelques années, dans le cercle très fermé des « 40 plus belles baies du monde», un club créé en 1996, à Londres, par trois membres du comité du tourisme du pays de Vannes, en Bretagne, et actuellement présidé par un ancien député du littoral picard. D'un côté, les panneaux publicitaires vantent, jusqu'au métro parisien, l'authenticité d'un écrin resté «sauvage»; de l'autre, une expression sonne comme un avertissement : «not'baie», manière de signifier qu'elle reste le domaine des locaux qui l'ont façonnée dès le Moyen Âge par un endiguement progressif et continu, les « renclôtures » (terme picard pour désigner les polders). Dans leur vocation première, les renclôtures constituent autant de grands travaux lancés par les seigneurs environnants pour faire avancer la terre sur la mer, dans un souci culturel au double sens du terme : culture de la terre, mais encore humaine. Les raisons profondes en sont, en effet, tout autant idéologiques qu'agronomiques, suivant par là le long mouvement civilisationnel de domestication de la nature par la soumission des éléments qu'inaugure la pensée cartésienne (Kalaora, 2010). Mais, dès la fin du XIX ${ }^{\mathrm{e}}$ siècle, cette destination agricole et pastorale se trouve concurrencée par le développement d'un mode de chasse qui consiste à se "hutter» au sein même des zones humides pour y traquer le gibier migrateur. La chasse à la «hutte» est née de cette immersion dans le biotope au travers d'une «mûche» (cachette). La technique des fleurons avait commencé à être contestée. Ces grands filets tendus à marée basse, où venaient se pendre les oiseaux, furent définitivement interdits avant la Première Guerre mondiale, considérés comme dangereux et cruels, alors qu'arrivaient les premiers flots de touristes (Estienne, 2001).

On ne peut nier que la pression cynégétique joua un rôle prééminent dans le recul progressif des usages agricoles du site, contribuant par là même à en faire une zone d'intérêt pour l'avifaune. C'est de cet héritage historique et plus ou moins mythifié dont se réclament les sauvaginiers contemporains pour se présenter en rempart à la mise en culture généralisée des zones humides, "sans quoi la baie serait devenue un immense champ de maïs». Pour eux, l'artificialisation des milieux naturels d'exception menace désormais ceux-ci par la «bétonisation», dont le touriste serait le vecteur. À l'appui de cette assertion, le complexe hôtelier de Belle Dune, au nord de l'estuaire, est régulièrement dénoncé. Destiné aux estivants fortunés, on lui reproche encore d'avoir englobé dans son emprise les équipements qui profitaient antérieurement à toute la population (Baticle et Mazzéi, 2011).

De même, toujours dans la limite septentrionale de l'aire considérée, la plus aboutie de ces emblématiques érections ex nihilo qui signent la mise en tourisme du territoire, à savoir le Parc ornithologique du Marquenterre (étymologiquement «la mer qui entre dans la terre»), fait figure de «Walt Disney des oiseaux» aux yeux des nemrods aguerris aux rigueurs des baies. Sa création, en 1973, répond dès le départ à un objectif d'accueil du public attiré par la faune sauvage (Jeanson, 1999), une tendance alors encore balbutiante dans la société française. Les huttes initiales sont désormais devenues des postes d'observation de l'avifaune pour les quelque 140000 touristes qui s'y pressent annuellement. Dans les années 1980, le mouflon fut introduit dans les dunes environnantes. Il ajoute, par sa présence atypique, une note d'exotisme qui suffit à discréditer, chez les résidents locaux, l'authenticité qui est pourtant le produit d'appel du parc.

À quelques encablures de là, un autre signe avertit le touriste qu'il entre dans une zone investie par d'autres usages que les siens et que l'on peine à classer dans une catégorie purement récréative. Sur le terre-plein central d'un vaste carrefour giratoire, domine en effet un "pied de hutte», soit la reconstitution miniature d'un marécage, au centre de laquelle trône une installation cynégétique déplacée pour les besoins de la démonstration. Ici, on entre dans «la patrie des sauvaginiers ", pour reprendre l'expression du président des chasseurs du département. Dans le même sens, ce n'est pas le fruit du hasard si ces sauvaginiers se sont opposés avec tant de virulence à l'édification d'un musée de la chasse, et ce, au nom d'un refus à l'endroit de toute vitrification de la pratique, considérée a contrario comme devant faire la démonstration de sa vitalité.

Sans multiplier davantage les exemples, insistons pour ce terrain sur l'antagonisme qui s'est élaboré, durant les 30 dernières années, entre la place initialement prééminente de la chasse et celle du tourisme, secteur désormais dominant sur le plan de la dynamique économique. Une rivalité qui ne s'expliquerait pas totalement sans le contexte de politisation avec, depuis 1989, une formation partisane, née justement en baie de Somme : Chasse, pêche, nature et traditions (CPNT). En ce sens, s'attarder sur le littoral picard détient l'avantage de poser son regard sur un espace où toutes les conditions semblent réunies pour que s'y développe un tourisme de chasse marchand : abondance du gibier, cadre juridique des associations gestionnaires, installations hôtelières et de restauration, autoroute à partir de 1998, propriétés foncières de l'État et des collectivités territoriales, difficultés à trouver des territoires de pratique. Il n'en est rien pourtant, les chasseurs locaux continuant à se transmettre les concessions de proche en proche, laissant pour seule ouverture des invitations sélectives qui répondent à deux critères : le localisme et l'entre-soi idéologique.

\section{Conclusion}

Pour évoquer les liens, en France, entre pratique cynégétique et tourisme, on peut partir de deux constats simples. Tout d'abord, on doit prendre en compte les contextes socio-économiques dans lesquels se déroulent ces luttes territoriales. Ce n'est pas un hasard si l'analyse s'est concentrée ici sur un site comme la côte picarde, un haut lieu de la nature patrimonialisée, mais également préemptée par des populations solidement implantées dans le temps, suspendues à la désindustrialisation et aux menaces de délocalisation (Baticle, 2003). L'écologie et le tourisme y sont ressentis négativement, parce qu'assimilés à des logiques externes d'expropriation et de captation de la dernière ressource considérée comme indéboulonnable : le territoire. Par cette appréhension, le tourisme est perçu comme un "mal nécessaire» (Baticle et Mazzéi, 2011) à cantonner autour des restaurants, mais à distance des huttes qui symbolisent une culture locale irrédentiste. Dans ce cadre, il n'est pas étonnant que le tourisme cynégétique y soit géré sur le mode de la cooptation. 
Ensuite, il convient d'envisager la spécificité du pratiquant dans ses représentations dominantes quant aux manières légitimes d'être chasseur. Le pérégrin qui se définit par sa quête du sauvage peine à se reconnaître dans l'appellation de «touriste». Ces deux variantes du déplacement relèvent de positionnements qui ont à voir avec la manière dont on conçoit le loisir, mais encore des stratégies distinctives antithétiques. La chasse, telle qu'elle s'autodésigne, entre dans ces activités qui résistent à l'idée d'une mise en tourisme. La signification donnée à la prédation n'y est pas étrangère, à savoir une "culture de la nature» qui aboutit au slogan «La nature est notre culture» (CPNT, 2001). En ce sens, les porteurs de fusils peuvent difficilement être rapprochés de la catégorie des consommateurs de nature. Cette difficulté ressort du mode de légitimation encore aujourd'hui dominant : la chasse reste ainsi pensée comme une relation socioterritoriale médiatisée par l'animal. Elle passe de ce fait par des procédures qui empruntent à l'inscription temporelle (l'autochtonie plus ou moins bricolée) ou au travail (l'aménagement de l'espace). Ceci s'explique par le fait d'une giboyeusité interprétée comme le produit d'une histoire collective dans la formation des biotopes et renouvelée par le labeur. Ces valeurs entrent alors en contradiction avec l'appropriation foncière du sol par la voie consumériste. Ces réalités prégnantes ne doivent pas se confondre avec une interprétation culturaliste, ou alors elles relèvent de cultures sociales : d'une part, celle des propriétaires qui ont aussi tout intérêt à conserver la maîtrise territoriale et, d'autre part, celle des sans terre qui voient un risque d'éviction derrière toute marchandisation de l'espace.

À la manière de Cassandre, c'est peut-être également que ces chasseurs ont compris l'enjeu intrinsèque contenu dans le tourisme, tel que le définit magistralement Philippe Bachimon (2006 : 15) : «forme de mobilité de loisir introduisant plus ou moins subrepticement du global dans le local». Leur marche se rapproche alors de ce que David Le Breton (2000 : 19) envisage dans l'acte de déambulation pédestre : une méthode de «réenchantement de la durée et de l'espace».

\section{Références}

ACTÉON (1998) Bilan final de l'étude de l'offre et de la demande cynégétique, Paris : La Documentation française, $34 \mathrm{p}$.

AUCANTE, Pierre et Joël MIRLOUP (1979) «Chasseurs et braconniers», DANS HESSE Jacques (sous la direction de), Sologne, Paris : BergerLevrault, 202 p.

AUCANTE, Marieke et Pierre AUCANTE (2004) Le livre du braconnier, Paris : Albin Michel, $191 \mathrm{p}$.

BACHIMON, Philippe (2006) «Territoire et identité. Un rapport ambigu», DANS FURT Jean-Marie et Franck MICHEL (sous la direction de), Tourismes et identités, Paris : L'Harmattan, p. 15-26.

BAGES, Robert et Jean-Yves NEVERS (1982) «L'organisation locale de la chasse. Autodéfense collective et régulation des conflits ", Études rurales, $\mathrm{n}^{\circ}$ 87-88, p. 209-221.

BARRAL, Pierre (1968) Les agrariens français, de Méline à Pisani, Paris : Armand Colin, $386 \mathrm{p}$.

BART, Jean (1988) «La conquête paysanne du droit de chasse sous la Révolution française », DANS VANNIER Paul et Daniel MEILLER (sous la direction de), L'imaginaire de la chasse : hier et demain, Le Creusot : Atelier CRC, p. 65-75.
BATICLE, Christophe (2003-2004) Chasse et environnement : implications réciproques? Étude cynégétique du site Natura 2000 «Estuaires et littoral picards» (tome $1: 2003$ ), "Arrière-littoral picard» (tome $2: 2004$ ), Amiens : UPJV (CEFRESS), 520 p. et 690 p.

BATICLE, Christophe (2005) Les chasseurs de la Somme : portrait social, Amiens : UPJV (Habiter), $180 \mathrm{p}$.

BATICLE, Christophe (2007) Les pratiques de chasse comme affirmations politiques $d u$ principe d'autochtonie : dimensions territoriales des luttes cynégétiques, thèse de doctorat en sociologie, Amiens : UPJV (CEFRESS), $989 \mathrm{p}$.

BATICLE, Christophe (2009) «L'espace socialisant de la mémoire familiale ou ce que chassent les chasseurs», DANS COCHARTCOSTE Dominique et Abel KOUVOUAMA (sous la direction de), Représentations et productions de l'espace dans les sociétés contemporaines, Paris : L'Harmattan, p. 147-201.

BATICLE, Christophe et Raphaël MAZZÉI (2011) Baie-Baie, cette mâ̂tresse. Habiter la nature au travers de la chasse, film documentaire, Amiens : Acsé («Atlas visuel picard »), 40?.

BECK, Corinne (1988) «Pratiques et enjeux de la chasse en Bourgogne au bas Moyen Âge», DANS VANNIER Paul et Daniel MEILLER (sous la direction de), L'imaginaire de la chasse : hier et demain, Le Creusot : Atelier CRC, p. 49-63.

BERTON, Antoine et David GAILLARDON (2013) La chasse, Paris : First, $448 \mathrm{p}$.

BOZON, Michel (1982) "Chasse, territoire, groupements de chasseurs", Études rurales, $\mathrm{n}^{\circ}$ 87-88: "La chasse et la cueillette aujourd'hui », p. 335-342.

BOZON, Michel, Jean-Claude CHAMBOREDON et Jean-Louis FABIANI (1981) «Les usages sociaux du cadre naturel. Élaboration sociale et conflits des modes de consommation de la campagne : l'exemple de la chasse», DANS Des arbres et des hommes, Le Paradou : Actes Sud, p. 273-279.

CHAMBOREDON, Jean-Claude (1982) «La diffusion de la chasse et la transformation des usages sociaux de l'espace rural», Études rurales, $n^{\circ}$ 87-88: «La chasse et la cueillette aujourd'hui», p. 233-260.

CHEVALLIER, Denis (sous la direction de) (2000) «Vives campagnes. Le patrimoine rural, projet de société», revue Autrement, n 194, 223 p.

RIPOLL, Fabrice et Sylvie TISSOT (2010) «Mobilité/autochtonie : sur la dimension spatiale des ressources sociales ", dossier de Regards sociologiques, $\mathrm{n}^{\circ} 40,109 \mathrm{p}$.

CORBIN, Alain (sous la direction de) (2009) L'avènement des loisirs. 18501960, Paris : Flammarion, 626 p.

CPNT - Chasse pêche nature et traditions (2001) Le manifeste de CPNT, Pau : CPNT, $65 \mathrm{p}$.

DALLA BERNARDINA, Sergio (2011) Retour du prédateur. Mises en scène du sauvage dans la société post-rurale, Rennes: PUR, $134 \mathrm{p}$.

DAUDET, Alphonse (1872) Les aventures prodigieuses de Tartarin de Tarascon, Paris : Payot, édition de 2007, 544 p.

DGCIS - Direction générale de la compétitivité, de l'industrie et des services (2012) Les chiffres clés du tourisme, Paris : ministère de l'Artisanat, du Commerce et du Tourisme, $6 \mathrm{p}$.

DURKHEIM, Émile (1894) Les règles de la méthode sociologique, Paris : PUF, édition de 2007, $144 \mathrm{p}$.

ESTIENNE, Jean (2001) «La chasse en baie de Somme», Bulletin de la Société d'histoire et d'archéologie de Saint-Valéry-sur-Somme, $\mathrm{n}^{\circ} 32$, p 2-9.

FACE - Fédération des associations de chasseurs en Europe (2010) Annual Report 2009-2010, Bruxelles : FACE, p. 16. 
GOETSCHMANN, Michel (2010) «Le tourisme de proximité», Les cahiers $d u$ CRISC, $\mathrm{n}^{\circ} 26$, p. 63-77.

HELL, Bertrand (1985) Entre chien et loup. Faits et dits de chasse dans la France de l'Est, Paris : Maison des sciences humaines et ministère de la Culture, $227 \mathrm{p}$.

HERVIEU, Bertrand et François PURSEIGLE (2013) Sociologie des mondes agricoles, Paris : Armand Colin, 318 p.

IFE - Institut français de l'environnement (1999) Les retombées économiques de la chasse en Sologne, Orléans : IFE, $140 \mathrm{p}$.

JEANSON, Claude (1999) La face cachée des dunes. Le domaine du Marquenterre, Saint-Quentin-en-Tourmont : Marcanterra, $191 \mathrm{p}$.

KALAORA, Bernard (2010) Rivages en devenir. Des horizons pour le Conservatoire du littoral, Paris : La Documentation française, $232 \mathrm{p}$.

LARRÈRE, Catherine (1988) «Jean-Jacques Rousseau : la forêt, le champ et le jardin », DANS CADORET Anne (sous la direction de), Chasser le naturel..., Paris : EHESS, p. 23-61.

LE BRETON, David (2000) Éloge de la marche, Paris : Métailié, 176 p.

LE GOFFE, Philippe et Dominique VOLLET (2008) «Économie et politique de la chasse : constats, analyses et idées pour l'action », colloque de la Société française d'économie rurale : Chasse, territoires et développement durable. Outils d'analyse, enjeux et perspectives, Clermont-Ferrand : Énitac, 20 p., <http://www.sfer.asso.fr/les_colloques2/les_colloques_passes/chasse_territoires_et_developpement_durable_outils_d_analyse_ enjeux_et_perspectives>, consulté le $1^{\mathrm{er}}$ septembre 2013.

LEJEUNE, Maxime (2005) Chasse, pouvoir, diplomatie, Chaumont : CrépinLeblond, $327 \mathrm{p}$.

LEROY-WARNIER, Louis et Armelle CARON (2008) «Le tourisme cynégétique. Enjeux d'un levier de développement local en devenir », colloque de la Société française d'économie rurale : Chasse, territoires et développement durable. Outils d'analyse, enjeux et perspectives, Clermont-Ferrand : Énitac, $11 \mathrm{p}$.

MAE - Ministère des Affaires étrangères (2003) Caractériser le tourisme responsable, Facteur de développement durable, rédigé par Alain Laurent, Paris : ministère des Affaires étrangères, $270 \mathrm{p}$.

MALAFOSSE DE, Jehan (1979) Droit de la chasse et protection de la nature, Paris : PUF, 482 p.

MARX, Karl (1850) Les luttes de classes en France, Paris : Éditions Sociales, édition de 1967, 218 p.

MISCHI, Julian (2008) «Les militants ouvriers de la chasse. Éléments sur le rapport à la politique des classes populaires », Politix, $\mathrm{n}^{\circ}$ 83, p. 133-153.

PATIER, Xavier (2002) La chasse, Paris : Le Cavalier bleu, 128 p.

PERRIER-CORNET, Philippe (sous la direction de) (2002) Repenser les campagnes, La Tour d'Aigues : Aube et DATAR, 279 p.
PINET, Jean-Michel (1987) L'économie de la chasse, Paris : Institut national agronomique, $93 \mathrm{p}$.

PINET, Jean-Michel (1993) Les chasseurs de France : organisation, typologie, économie, horizon 2000, Paris : Institut national agronomique, 103 p.

RENAHY, Nicolas (2005) Les gars du coin. Enquête sur une jeunesse rurale, Paris : La Découverte, 284 p.

RENAHY, Nicolas (2010) «Classes populaires et capital d'autochtonie. Genèse et usages d'une notion", Regards sociologiques, $\mathrm{n}^{\circ} 40$ : «Mobilitél autochtonie : sur la dimension spatiale des ressources sociales», p. 9-26.

RETIÈRE, Jean-Noël (2003) «Autour de l'autochtonie. Réflexions sur la notion de capital social populaire ", Politix, n 63, p. 121-143.

ROBERT, Michel (1986) Sociologie rurale, Paris : PUF, 125 p.

SALESSE, Dominique (1993) «Vous avez dit louphoque?», Le siffleur de la baie de Somme, revue de l'ACDMP baie de Somme, $\mathrm{n}^{\circ}$ 8, p. 14.

SALVADORI, Philippe (1996) La chasse sous l'Ancien Régime, Paris : Fayard, $462 \mathrm{p}$.

SANNIER, Lancelot (2004) À qui appartient la baie de Somme?, mémoire de maîtrise en géographie, Amiens : UPJV, 123 p.

SCALLAN, David (2008) «Neglected Economic Opportunities : What Place for Hunting in Rural Ireland?", colloque de la Société française d'économie rurale : Chasse, territoires et développement durable. Outils d'analyse, enjeux et perspectives, Clermont-Ferrand : Énitac, 16 p.

TESTART, Alain (1988) «La chasse préhistorique : mythes et réalités », DANS VANNIER Paul et Daniel MEILLER (sous la direction de), L'imaginaire de la chasse : hier et demain, Le Creusot: Atelier CRC, p. 21-29.

UNIVERS NATURE (2009) «Comparatif sur la chasse aux oiseaux, en Europe»,

<http://www.nature46.fr/LaNature/Informations/InformationsGenerales/ ChasseursEurope.htm>, consulté le 30 août 2013.

VALICOURT de, Joseph (1972) «L'avenir de la chasse en Picardie», Picardie information : revue d'information économique, $\mathrm{n}^{\circ} 8$, p. 43-46.

VIVENT, Céline (2005) CPNT : entre écologisme et poujadisme? Socioanthropologie d'un mouvement des campagnes, Paris : L'Harmattan, 169 p.

VOLLET, Dominique et Geneviève BRETIÈRE (2008) «La chasse : quel impact sur l'emploi local?", colloque de la Société française d'économie rurale : Chasse, territoires et développement durable. Outils d'analyse, enjeux et perspectives, Clermont-Ferrand : Énitac, 22 p.

WIDMER, Jean-Paul (2008) «Compte-rendu d'expérience. Actéon, un outil d'intégration des activités cynégétiques dans le développement durable des territoires », colloque de la Société française d'économie rurale : Chasse, territoires et développement durable. Outils d'analyse, enjeux et perspectives, Clermont-Ferrand : Énitac, 8 p. 